\title{
Skin blood flow changes during apneic spells in preterm infants
}

\author{
H.E. Suichies ${ }^{a}$, J.G. Aarnoudse ${ }^{a}$, A. Okken ${ }^{a}$, H.W. Jentink ${ }^{b}$, F.F.M. \\ de Mulb and J. Greve ${ }^{b}$ \\ ${ }^{a}$ Department of Obstetrics and Pediatrics, University Hospital Groningen, Oostersingel 59, 9713 EZ \\ Groningen, and 'Department of Applied Physics, Twente University of Technology, P.O. Box 217, 7500 \\ AE Enschede (The Netherlands)
}

Accepted for publication 1 May 1989

\section{Summary}

Changes in skin blood flow during apneic spells were determined in 18 preterm infants using a diode laser Doppler flow meter without light conducting fibres. Heart rate, nasal air flow, impedance pneumography, skin and incubator temperature and laser Doppler skin blood flow were recorded simultaneously in each infant. During 212 apneic spells with a duration of $11.6 \pm 7.5 \mathrm{~s}$ (mean \pm S.D.) (range 6.0 $-48.0 \mathrm{~s}$ ), the laser Doppler skin blood flow was measured. In all children except one, the majority of the apneic spells was associated with a decrease in skin blood flow. During 155 apneic spells $(\mathbf{7 3 \%})$ skin blood flow decreased significantly $P<$ 0.025 ), the maximum decrease being $16.7 \pm 14.8 \%, 28.5 \pm 23.9 \%$ and $18.9 \pm 16.1 \%$ (mean \pm S.D.) for central, obstructive and mixed apneic spells, respectively. The decrease in skin blood flow started immediately after the beginning of apneic spells in $71 \%$, the rest started with a mean delay of $3.4 \mathrm{~s}$ (range $0.1-7.0$ s). No relation was found between the decrease in skin blood flow and the duration of the apneic spells. Thirty-four percent of the apneic spells were accompanied by bradycardia. In apneic spells accompanied by bradycardia the decrease in skin blood flow was not related to the fall in heart rate.

skin blood flow; laser Doppler; apnea; apneic spells; bradycardia; preterm infant

Correspondence to: Professor J.G. Aarnoudse, Department of Obstetrics, University Hospital Groningen, Oostersingel 59, 9713 EZ Groningen, The Netherlands. 


\section{Introduction}

Cardiovascular changes related to apneic spells have been reported by several authors. Changes in heart rate [3,4], blood pressure [4], pulse pressure [4] and cerebral blood flow [9] have been described during apnea in newborns. Little is known, however, about what happens with peripheral blood flow during apneic spells in the newborn.

Redistribution of blood flow in favour of vital organs such as the brain is likely to influence the circulation in other organs, for instance the skin. Storr described a decrease in limb blood flow during apneic episodes with bradycardia in preterm infants [13]. In that study blood flow was measured by venous occlusion plethysmography and limb blood flow recordings were only made during long-lasting apnea. New techniques such as laser Doppler flowmetry enables us now to measure fast, reliably and continuously the newborns skin blood flow. Therefore a study was undertaken to investigate: (1) if changes in skin blood flow do occur during apneic spells in newborn infants; (2) how fast these changes occur during apneic spells; and (3) if skin blood flow changes were dependent on the origin of apneic spells.

\section{Infants and methods}

We studied changes in skin blood flow during apneic spells in 18 preterm infants. Infants were born at a mean gestational age of 30.7 weeks (range 26-36 weeks) with a mean birth weight of $1365 \mathrm{~g}$ (range $720-2250 \mathrm{~g}$ ). Nine infants were small for gestational age (birth weight $<\mathrm{P}_{10}$ ). Ultrasound examination revealed cerebral hemorrhage in 4 infants. Respiratory pathologies such as hyaline membrane disease (HMD), broncho pulmonary dysplasia (BPD), or respiratory distress syndrome (RDS), were present in 9 infants. The clinical data of the infants are summarized in Table I. Measurements were performed in incubators with all infants in thermoneutral condition. Recordings lasted for a minimum of $1 \mathrm{~h}$ following a stabilisation period of $10-15 \mathrm{~min}$. During recordings eye condition and presence and type of general movements were observed. The observations and the respiratory pattern were used to determine the behavioral state of the infants, according to the criteria of Prechtl [10].

Skin blood flow was measured on the forehead in 15 infants and on the upper leg in 3 infants in accordance with the laser Doppler method described by others $[8,12,15]$. In short, this method is based on the following principle. Coherent laser light, illuminating the skin, is scattered by static skin structures and moving particles, e.g. red blood cells. Scattered light from a moving particle, however, undergoes a frequency shift (Doppler principle). Backscattered Doppler shifted and unshifted light, mixed on a photodetector surface produce a frequency spectrum of beat notes, which is dependent on the mean red blood cell velocity and the number of red blood cells. A differential detector system is used, and signal processing between $30 \mathrm{~Hz}$ and $30 \mathrm{kHz}$ provides an output in volts, proportional to blood flow.

We used a diode laser Doppler flow meter as previously reported [14]. This laser Doppler flow meter has a laser diode (Matsusita, $3 \mathrm{~mW}$ output at a wave length of $790 \mathrm{nM}$ ) and the 2 photodiodes (BPX-40) integrated in the probe. The weight of the 


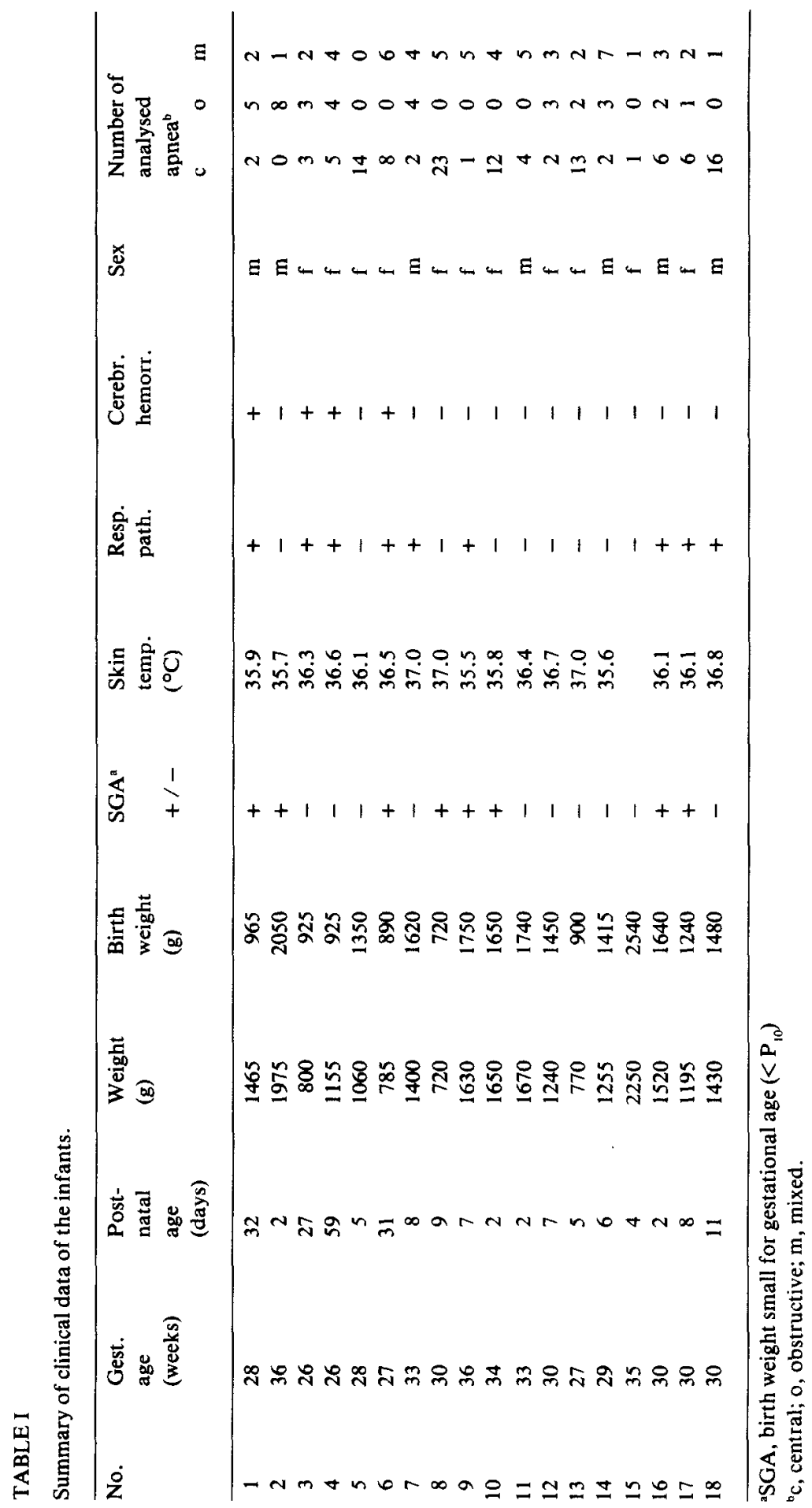


probe is $\mathbf{4} \mathbf{g}$ and it is attached to the skin with double-sided adhesive tape. In addition to the laser Doppler signal the following data were recorded using a multichannel Beckmann strip chart recorder and a Teac MR-30 cassette recorder: skin temperature (Yellow Springs thermistor probe 409-B), heart rate and respiratory wave forms. The latter were obtained via both thoracic impedance and a thermistor probe positioned in front of the nose.

Apneic spell was defined as the cessation of breathing for a minimum of $6 \mathrm{~s}$ [7]. Apneic spells were classified as central, obstructive or mixed apneic spells [5]. A total of 590 apneic spells were observed, whose origin could be divided into $45 \%$ central, $34 \%$ obstructive and $21 \%$ mixed apneic spells. A total of 212 apneic spells were analysed, the others being excluded on account of movement artefacts in the laser Doppler tracings or in the impedance pneumography tracings. The duration of each apneic spell was measured. (To avoid severe hypoxia 3 apneic spells were terminated artificially by tactile stimulation because of their long duration.)

Bradycardia was defined as a decrease in heart rate of more than $5 \%$ of the mean heart rate, measured for $1 \mathrm{~min}$ prior to the onset of apneic spells.

Skin blood flow values are given in volts. The zero level of the laser Doppler flow signal was determined by local occlusion of the vascular bed. This could be achieved by pushing the probe against the skull or occluding the arteria femoralis. The skin blood flow changes during apneic spells are expressed as the percentage of decrease. This percentage decrease was calculated by comparing the minimum flow values for 1 min prior to and during apneic spells. Skin blood flow changes within one standard deviation (S.D.) of the flow signal $1 \mathrm{~min}$ prior to apneic spells were designated as no change.

All data recorded on tape were fed into a microcomputer at a sample frequency of $10 \mathrm{~Hz}$. For statistical analysis the $t$-test for paired samples, Pearson's cross correlation test, and the Chi-square test were used. A $P$ value of 0.05 was taken as level of significance.

\section{Results}

Representative recordings of skin blood flow changes during central, obstructive and mixed apneic spells, with a concomitant bradycardia, are given in Fig. 1.

\section{Skin blood flow changes}

In all children except one the majority of the apneic spells was associated with a decrease in skin blood flow. Skin blood flow decreased significantly in $155(73 \%)$ of the 212 apneic spells analysed. This decrease in skin blood flow was observed in $68 \%$ of the infants with central apneic spells, in $80 \%$ with obstructive apneic spells and in $77 \%$ with apneic spells of mixed origin. The average decrease in skin blood flow in these 155 apneic spells was $16.7 \%, 28.5 \%$ and $18.9 \%$ for central, obstructive and mixed apneic spells, respectively, the differences in decrease between the 3 types of apnea being significant $(P<0.01)$. An increase in skin blood flow was seen during 15 apneic spells $(6.2 \%)$ in 9 infants. The decrease in skin blood flow started simultaneously with the onset of an apneic spell in $71 \%$. For the remaining apneic 


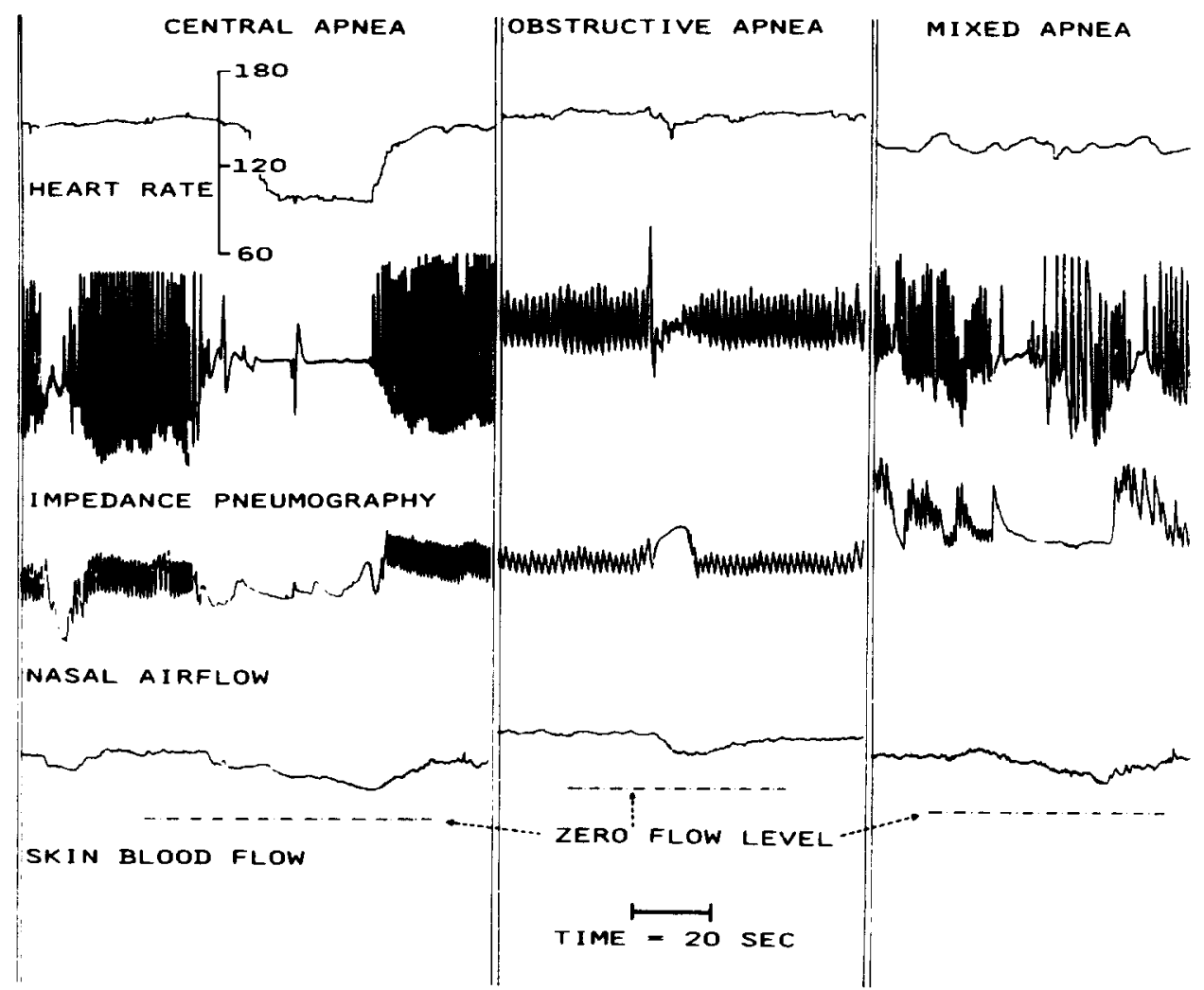

Fig. 1. Representative recordings of heart rate, impedance pneumography, nasal airflow and skin blood flow during central $(2 \times)$, obstructive and mixed apneic spells.

spells, the onset of skin blood flow decrease was delayed, the mean delay being $3.4 \mathrm{~s}$ (range 0.1-7.6 s).

The mean duration of an apneic spell was $10.3,10.2$ and $14.3 \mathrm{~s}$ for central, obstructive and mixed apneic spells, respectively. There was no relation between the duration of apneic spells and the degree of skin blood flow decrease for the 3 types of apneic spells (Table II).

\section{TABLE II}

Correlation coefficients in three types of apneic spells on maximum $\%$ flow decrease, $\%$ of bradycardia and duration of apneic spells

\begin{tabular}{llll}
\hline Correlation coeff. between: & Central & Obstructive & Mixed \\
\hline Max \% flow decr. - apnea duration & 0.22 & 0.16 & 0.32 \\
Max \% flow decr. - \% bradyc. & $0.31^{*}$ & 0.57 & $0.63^{* *}$ \\
Apnea duration - \% bradyc. & $0.65^{* *}$ & 0.51 & $0.56^{*}$
\end{tabular}

${ }^{*} P<0.05$

$* * P<0.01$ 


\section{Apnea and bradycardia}

Seventy-three $(34 \%)$ of the 212 apneic spells analysed were accompanied by a bradycardia (Table III). The incidence of reduced skin blood flow during apneic spells was higher when bradycardia was present $(P<0.01$, Chi-square test), although the difference was small. The onset of bradycardia started simultaneously with the beginning of an apneic period in $22 \%$, and with a mean delay of $5.7 \pm 2.3 \mathrm{~s}$ (range $1.0-12.0 \mathrm{~s}$ ) in $78 \%$. The degree of bradycardia was slightly correlated with the maximum percentage of flow decrease for mixed apneic spells (Table II).

\section{Behavioral states}

The behavioral state of the premterm infants could be determined in 55 of the 212 apneic spells. Twenty-four apneic spells were seen during quiet sleep and 31 during active sleep. In the others the behavioral state could not be determined. For mixed apneic spells during quiet sleep, the mean skin blood flow decrease was $16.8 \pm 13.1 \%$ (mean \pm S.D.), whereas during active sleep it was $30.2 \pm 13.8 \%$ (mean \pm S.D.), the difference being statistically significant $(P=0.05)$.

\section{Other factors}

The amount of skin blood flow decrease during apneic spells was not related to the following conditions: small for gestational age, HMD, BPD, RDS and cerebral hemorrhage.

\section{Discussion}

The results of the present study indicate that during the majority of apneic spells a considerable decrease in skin blood flow occurs which is independent of the presence of bradycardia. To our knowledge, there have not been any previous reports on changes in skin blood flow associated with apneic spells. Cardiovascular changes associated with apneic episodes in preterm infants have been reported by several authors $[3,4,9,13]$. Storrs [13], who measured cardiovascular reflex responses by means of venous occlusion plethysmography during apnea in preterm infants, found a decrease in the blood flow of the peripheral limb in all the apneic episodes studied. The average decrease in limb blood flow was $43 \pm 20 \%$ (range 6.1-85\%). The drop in limb blood flow was significantly correlated to bradycardia $(r=0.509)$.

TABLE III

Number of apneic spells with or without concomitant bradycardia and with or without skin blood flow decrease

\begin{tabular}{lccc}
\hline Apneic spells & With flow decrease & Without flow decrease & Total \\
\hline With bradycardia & 63 & 10 & 73 \\
Without bradycardia & 92 & 47 & 139 \\
Total & 155 & 57 & 212 \\
\hline
\end{tabular}

$\chi^{2}=9.85, P=0.002$ 
In that study, however, only "apneic episodes lasting long enough to produce cardiovascular changes" were analysed. Angel-James and Daly [1] reported cardiovascular responses resulting in bradycardia and systemic vasoconstriction during apnoeic hypoxia in anaesthetized dogs. Their studies have shown that the cardiovascular responses during apneic episodes are, at least in part, the result of primary reflexes from carotid and aortic body chemoreceptors activated by hypoxemia and hypercapnia following apnea. These reflexes include an increase in systemic vascular resistance on account of vasoconstriction in non-vital tissues and organs. If the cardiac output can be maintained, redistribution of blood flow takes place in favor of vital organs, such as the brain.

Until now it was not clear whether the peripheral vasoconstriction closely follows the apneic spells or coincides with it. The short $(0.025 \mathrm{~s})$ response time of the laser Doppler system allowed us to accurately determine the changes in skin blood flow in relation to apneic spells. It was found that the decrease in skin blood flow virtually coincided with the onset of apneic spells in the majority of the apneic spells studied. Similar, fast responses in skin blood flow were observed during periodical breathing (Fig. 2) in association with apneic spells [11]. These findings suggest either

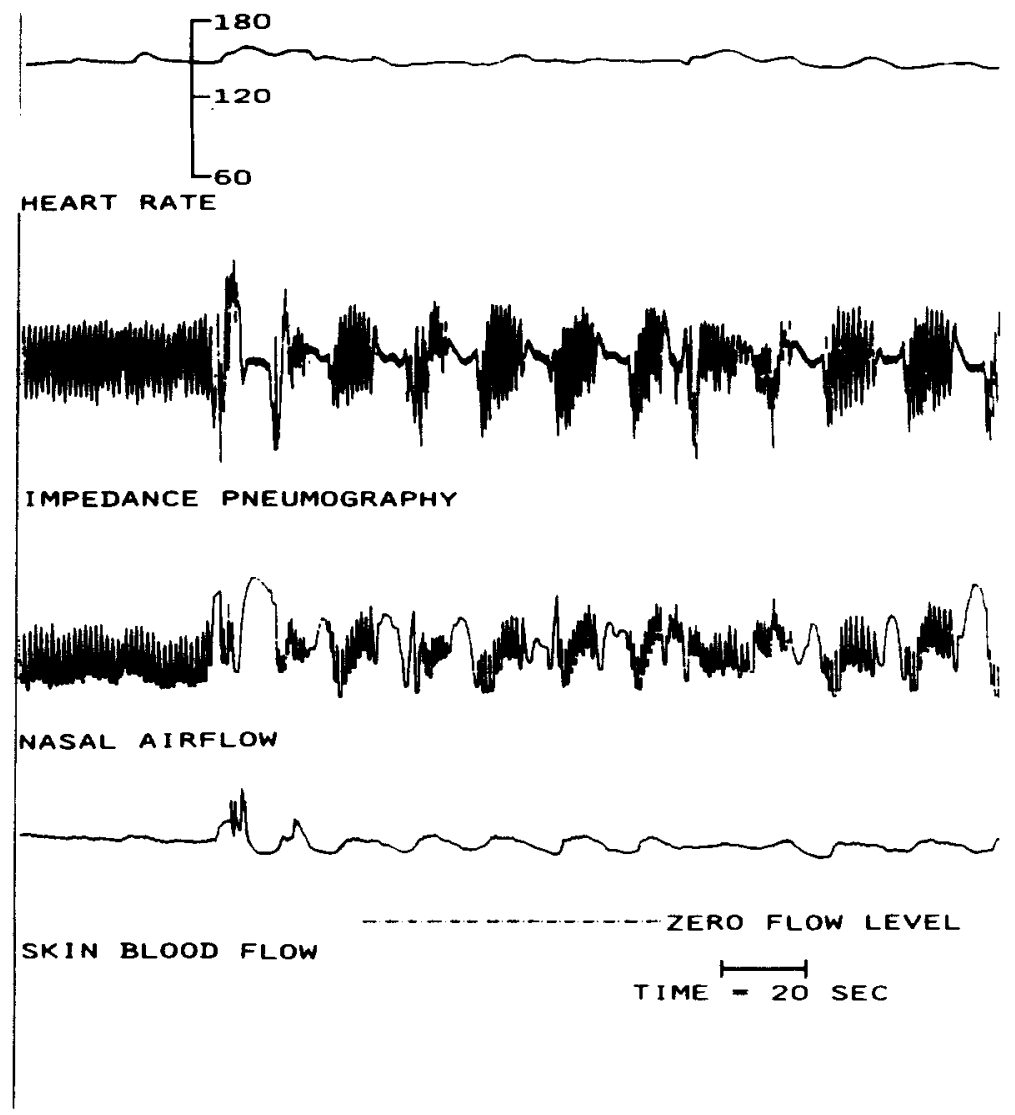

Fig. 2. Recording of skin blood flow during periodic breathing. 
a common cause for apneic spells and skin vasoconstriction or very rapid reflex vasoconstriction. The fact that both the length of apneic spells and the degree of bradycardia did not influence the amount of flow decrease, supports this idea.

The variability in the amount of flow decrease and the time delay between the onset of apneic spells and the beginning of the flow decrease indicates the existence of interfering mechanisms. We have no data on the state of lung inflation at the onset of apneic spells, but the more or less inhibitory effect of a pulmonary vagal inflation reflex [1] might be an explanation for this variability. Another possible explanation is the behavioral state of the infant. During active sleep in mixed apneic spells, the skin blood flow decrease was found to be significantly greater than during quiet sleep. It has been shown that during active sleep there is increased and more variable sympathetic activity [6]. Due to the predominant role of the sympathetic system in the control of skin blood flow, this will result in more vasoconstriction in the skin. Our data on the behavioral state during central and obstructive apneic spells were not sufficient to establish differences. A remarkable finding was the considerably greater reduction in skin blood flow in obstructive apneic spells as compared to apneic spells of central origin. Obstructive apneic spell is probably a more stressing event than central apneic spell and may therefore result in a greater increase in sympathetic activity and the release of vasoactive hormones causing increased vasoconstriction.

From this study it can be concluded that: (1) forehead skin blood flow, measured by the laser Doppler method, decreases significantly during apneic spells in preterm infants; (2) the onset of the decrease in skin blood flow mostly coincides with the onset of apnea; and (3) the decrease in skin blood flow is not related to bradycardia.

\section{Speculation}

Regarding the predominant role of sympathetic activity on skin blood flow it can be assumed that the fall in skin blood flow is due to an increased sympathetic activity. The latter might be caused by a reflectory mechanism as described by Cordero [2]. Therefore, further research on the relationship between skin blood flow, sympathetic activity and apnea is required.

\section{Acknowledgements}

The authors are grateful to Professor H.F.R. Prechtl for reviewing this manuscript. This study was supported by the Netherlands Technology Foundation (S.T.W.)

\section{References}

1 Angel James, J.E. and de Burgh Daly, M. (1969): Cardiovascular responses in apnoeic asphyxia: role of arterial chemoreceptors and the modification of their effects by a pulmonary vagal inflation reflex. J. Physiol., 201, 87-104.

2 Cordero, L. and Hon, E. (1971): Neonatal bradycardia following nasopharyngeal stimulation. J. Pediatr., 78, 441. 
3 Daily, W.J.R., Marshall, K. and Meyer, H.B.P.(1969): Apnea in premature infants: monitoring, incidence, heart rate changes and an effect of environmental temperature. Pediatrics, 43, 510-518.

4 Girling, D.J. (1972): Changes in heart rate, blood pressure and pulse pressure during apnoeic attacks in newborn babies. Arch. Dis. Childh., 47, 405-410.

5 Guilleminault, C., Tilkian, A. and Dement, W.C. (1976): The sleep apnea syndromes. Annu. Rev. Med., 27, 465.

6 Hagbarth K.E., Hallin R.G., Hongell A., Torebjörk, H.E. and Wallin, B.G. (1972): General characteristics of sympathetic activity in human skin nerves. Acta Physiol. Scand., 84: 164-176.

7 Hoppenbrouwers, T., Hodgman, J.E., Harper, R.M., Hofmann, E., Sterman, M.B. and McGinty, D.J. (1977): Polygraphic studies of normal infants during the first six months of life: III. Incidence of apnea and periodic breathing. Pediatrics, 60, 418-425.

8 Nillson, G.E., Tenland, T. and Oberg, P.A. (1980): Evaluation of a laser Doppler flow meter for measurement of tissue blood flow. IEEE Trans. Biomed. Eng., 27, 12-19.

9 Perlman, J.M. and Volpe, J.J. (1985): Episodes of apnea and bradycardia in the preterm newborn: impact on cerebral circulation. Pediatrics, 76, 333-338.

10 Prechtl, H.F.R. (1974): The behavioral states of the newborn infant (a review). Brain Res., 76: 185 -212 .

11 Rigatto, H. and Brady, J.P. (1972): Periodic breathing and apnea in preterm infants. I. Evidence for hypoventilation possibly due to central respiratory depression. Pediatrics, 50, 202-218.

12 Stern, M.D., Lappe, D.L., Bowen, P.D. et al. (1977): Continuous measurement of tissue blood flow by laser Doppler spectroscopy. Am. J. Physiol., 232, 441-448.

13 Storrs, C.N. (1977): Cardiovascular effects of apnea in preterm infants. Arch. Dis. Child, 52, 534 -540 .

14 Suichies, H.E., Aarnoudse, J.G., Okken, A., Jentink, H.W., de Mul, F.F.M. and Greve, J. (1987): Forehead skin blood flow in normal neonates during active and quiet sleep, measured with a diode laser Doppler instrument. Acta Paediatr. Scand., 77, 220-225.

15 Wunderlich, R.W., Folger, R.L., Giddon, D.B. and Ware, B.R. (1980): Laser Doppler blood flow meter and optical plethysmograph. Rev. Sci. Instrum., 51(9), 1258-1262. 